\title{
Neuropsychological and psychiatric complications in endoscopic third ventriculostomy: a clinical
} case report

\author{
A Benabarre, J Ibáñez, T Boget, J Obiols, A Martínez-Aran, E Vieta
}

\begin{abstract}
The clinical case report of a patient who underwent an endoscopic third ventriculostomy for the treatment of a slit ventricle syndrome is presented. After surgery the patient developed a severe complication consisting of an organic personality disorder, characterised by impulsiveness, physical heteroaggressiveness, binge eating, hypersomnia and impairment of memory, and frontal-executive functions. A frontal lobe lesion may explain some of the symptoms presented, such as the uncontrolled impulses, the aggressive behaviour, and even the binge eating. However, a longitudinal neuropsychological evaluation showed a severe deficit in immediate memory and difficulties in planning and consolidation of newly learned information, which may be best related to damage in the frontal basal structures of the brain: the fornix and its connection to the hippocampus and the mamillary bodies. Postoperative MR images confirmed the clinical hypothesis. The emergence of such a severe organic personality disorder and cognitive disturbances as a psychiatric complication of an endoscopic third ventriculostomy has not, it seems, been previously reported elsewhere. Clinicians should take these possible complications into account when recommending this so-called minimally invasive neuroendoscopic procedure. (F Neurol Neurosurg Psychiatry 2001;71:268-271)
\end{abstract}

Keywords: third ventriculostomy; neuropsychological complications; neuroendoscopy

Changes in personality defined as organic personality disorders, which may occur after the administration of noxious organic agents, account for $2 \%-5 \%$ of all personality disorders. These disorders should be categorised as"disorders of the personality or behaviour owing to illness, cerebral damage or dysfunction" (CIE-10), ${ }^{1}$ or "change of personality owing to... - specifying the nosologic agent responsible" (DSM-IV). ${ }^{2}$

The clinical picture of such a disorder generally starts with a marked change in lifestyle and the emergence of traits of an unadjusted personality, after the necessary previous presence of an organic causal factor and, always, with a previously normal behaviour pattern.

There are many causes that could lead to organic personality disorders. The most frequent is head injury, but also many cases have been reported in the literature related to stroke, brain tumours-especially those affecting frontal or temporal lobes-epilepsy, Huntington's disease, multiple sclerosis, toxic drugs, neurosyphilis, HIV infection, and also surgery. ${ }^{3}$

\section{Case report}

Our patient was a 20 year old man diagnosed with a slit ventricle syndrome. At the age of 8 months, he underwent shunt surgery for a non-communicating hydrocephalus due to aqueductal stenosis. Over the years he developed multiple complications of the CSF shunt system, including several obstructions and infections, which led to 13 surgical revisions. Although neuropsychological explorations were not performed, both the patient and the family deny the existence of memory difficulties before surgery. The current episode began with severe headaches, nausea, and vomiting in the presence of slit ventricles on CT and an increased CSF pressure recorded by means of continuous lumbar pressure monitoring $(>30$ $\mathrm{mm} \mathrm{Hg}$ ). After initial conservative treatment, improvement of the patient's condition was not considered satisfactory and an endoscopic third ventriculostomy with shunt removal was proposed.

On 21 January 1997 the patient underwent surgery under general anaesthesia, and through a right side preocoronal burr hole, a $6.5 \mathrm{~mm}$ rigid neuroendoscope (Gaab Endoscope, Storz, Tutlingen, Germany) was inserted in the frontal horn of the right lateral ventricle, following the technique described by Vries ${ }^{4}$ and Vries et al..$^{5}$ Fenestration of the floor of the third ventricle to the basal cisterns was performed with bipolar coagulation and enlarged with a $3 \mathrm{~F}$ Fogarty catheter. The night before surgery the shunt system was removed and external ventricular drainage placed to obtain preoperative control of the ventricular 
size. However, two events greatly hindered surgery. Firstly, the resistance of the ventricular wall to puncture was much greater than in other similar procedures and, secondly, despite closing the external ventricular drainage 6 hours before surgery, the ventricular system was extremely small, and the movements of the endoscope, mainly to achieve access to the third ventricle through Monro's foramen and also movements into the third ventricle, were greatly hampered. After performing the third ventriculostomy, the endoscope was removed, and damage to the right fornix was noted.

The postoperative course was almost uneventful. Clinical symptoms related to high intracranial pressure improved dramatically. Postoperative cine-MRI demonstrated the patency of the ventriculostomy and only a slight increase in the size of the ventricular system was appreciated on the follow up CT and MRI studies.

Only a moderate impairment in immediate memory was noted on discharge. Based on previous experience, this defect was considered to improve during the subsequent months.

Three months after surgery the first neuropsychological studies were completed. They showed obvious deficits in immediate memory tasks. It was ascertained that his intellectual ability in the subtests of vocabulary, block design, and similarities of the Wechsler adult intelligence scale (WAIS) ${ }^{6}$ was in the lower average normality. There was a deficit in immediate memory according to logical memory and visual reproduction (Wechsler memory scale-revised). ${ }^{7}$ A reduced receptive span (digit span) ${ }^{6}$ and impairment in frontalexecutive function (trail making test of the Halstead-Reitan battery $)^{8}$ was also found, although the most affected function was that of delayed recall (logical memory II and visual reproduction $\mathrm{II}){ }^{\text {? }}$

Seven months after surgery the patient was admitted to the endocrinological department because of progressive alterations in his eating behaviour, which included episodes of binge eating with no sign of satiation, with a consequent increase in weight (about $25 \mathrm{~kg}$ ). Hypersomnia was notable, as he slept 13 hours a day and, for the first time, his family reported a change in behaviour consisting of greater irritability, aggressiveness, and attention disturbance. He was diagnosed with an impulse control disorder and treated with $40 \mathrm{mg}$ fluoxetine

Table 1 Neuropsychological score profile in $T$ notes

\begin{tabular}{lll}
\hline & $\begin{array}{l}\text { Three months after } \\
\text { intervention }\end{array}$ & $\begin{array}{l}\text { Seven months after } \\
\text { intervention }\end{array}$ \\
\hline Intellectual ability: & & 50 \\
$\quad$ Vocabulary & 47 & 47 \\
$\quad$ Block design & 43 & 50 \\
$\quad$ Similarities & 50 & 37 \\
Memory: & 30 & 47 \\
$\quad$ Digit span & 43 & 43 \\
$\quad$ Logical memory I & 30 & 37 \\
$\quad$ Visual reproduction I & 30 & 33 \\
Verbal paired associates & 30 & 23 \\
$\quad$ Logical memory II & 20 & 30 \\
$\quad$ Visual reproduction II & 30 & 33 \\
Frontal-executive: & 33 & \\
$\quad$ Trail making A & & \\
Trail making B & & \\
\hline
\end{tabular}

daily. The treatment was partially effective. The number and intensity of binge eating episodes diminished, but his weight, sleeping rhythm, and behavioural disorder did not change. He became progressively more irritable and aggressive, a fact which distressed his family, who had never seen him act this way before. His episodes of impulsiveness increased in frequency and intensity, greatly impairing familial functioning. Memory deficits became obvious and he was afraid to leave home because "he couldn't remember how to get back". He also showed lack of motivation and apropositivism.

Eight months after surgery, he was admitted to the psychiatric department of the same hospital after an episode of physical aggressiveness. At that time, he had an average of one episode of aggressiveness a day. Physical examination showed the presence of wine grooves in the axiliary and inguinal region and mild gynaecomastia. Neurological examination was normal. Brain CT showed a moderate increase in size of the ventricular system without signs of active hydrocephalus. Asymmetry in the size of the lateral ventricles was seen. Brain MRI confirmed the CT findings and showed the path of the endoscope through the right frontal lobe. Cerebral SPECT with ${ }^{99 \mathrm{~m}}$ Tc-HMPAO did not detect any regional perfusion deficits in the cerebral cortex and the EEG was normal.

Seven months later, the patient again underwent evaluation with neuropsychological tests. Slowed frontal-executive functions (self programming and planning) persisted, both associated with frontal lobe dysfunction. In the memory area, there was measurable improvement in the immediate memory but a severe deterioration in the delayed recall persisted, with a loss in verbal material and visual information processing (table 1). Evident emotional instability with a tendency to extroversion and difficulty in controlling impulses was noted in the personality area. The symptomatic profile showed measurable levels of anxiety and hypersensitivity in his relationships with others. He showed difficulty in self control and had a low tolerance to frustration, which could give rise to badly formulated hostile reactions, but psychotic symptoms were not found.

Treatment with carbamazepine was started at a dose of $500 \mathrm{mg} /$ day and the treatment with $40 \mathrm{mg}$ fluoxetine daily was continued. During his stay in hospital no episode of aggressiveness was seen and he was discharged after 3 weeks, with the diagnosis of personality disorder owing to his illness, injury, or an unspecified cerebral dysfunction. Seven months after discharge, no episode of physical aggression was reported. Nevertheless, some irritability, severe memory loss, and hypersomnia persisted, although the binge eating has reduced in intensity.

\section{Discussion}

Organic personality disorders have classically been associated with head injury. ${ }^{9-11}$ In the literature there are many reports on patients with organic personality disorders presenting with a frontal syndrome: lack of inhibition, lack 

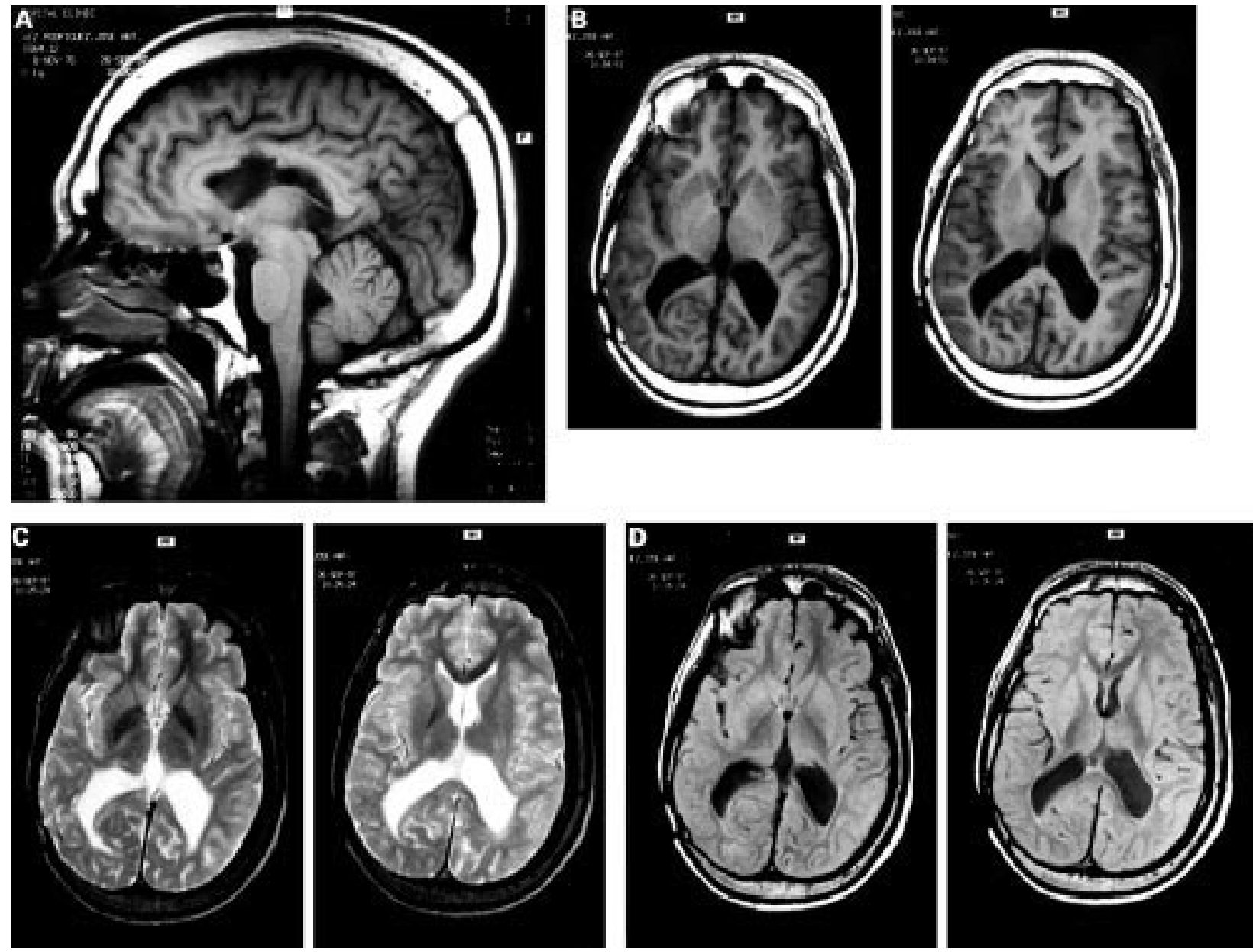

Figure 1 Images suggesting hypothalamic lesions: (A) sagittal plane, (B) T1 images, (C) T2 images, and (D) protonic density images.

of control over impulses, inappropriate mood, aggressiveness, and others, ${ }^{11}$ which, in most patients, are due to tumours, cerebral vascular events, or trauma. In step with scientific advances, more changes in personality have progressively been related to outside agents.

Endoscopic third ventriculostomy has been described as an effective technique for the treatment of some cases of hydrocephalus and seems to be specially appropriate for patients with aqueductal stenosis. Some complications of this technique have been described beforenamely, transitory herniation syndrome, basilar artery lesion, ventriculitis, decrease in the level of consciousness, transitory hemiparesis, and others. ${ }^{12}$ To the best of our knowledge, no case of such a severe postventriculostomy psychiatric or neuropsychological complication has been published. ${ }^{1}$

The ventricular approach of endoscopic neurosurgery is almost always visible via structural neuroimaging techniques. Thus, the area of postoperative malacia after the trajectory of the rigid endoscope through the frontal lobe and corpus callosum could not be related to the postoperative clinical findings in this patient. On the other hand, deficits detected through neuropsychological longitudinal evaluations were most appropiately associated with injuries in other neural structures situated close to the area of surgical intervention, such as the fornix and the hypothalamus. Those lesions were detected both during the surgical procedure and on the postoperative MRI (fig 1).

Although injuries of the right frontal lobe may explain some of the symptoms of the organic personality disorder (difficulties in sustaining attention, incapacity to plan, high level of impulsiveness, lack of inhibition, poor motivation, and other compulsive symptoms), considering the alterations shown in the longitudinal neuropsychological tests, where our patient showed a very measurable deficit in delayed recall in the medium term (anterograde amnesia), the surgical and neuroimaging findings, and the previous experience in similar cases, we think that other explanations could be more reasonable.

Thus a severely reduced receptive span was found, which did not allow integration or fixation of information in a logical context, which, in turn, caused many difficulties in consolidating verbal paired associates learning. ${ }^{7}$ This neuropsychological pattern is characteristic of dysfunctions of the hippocampal nuclei; a supposed injury of the fornix may explain this problem area, given that it corresponds functionally with the main hippocampus efferent, directly associated with the mamillary bodies. ${ }^{14}$ None the less, the postsurgical performance showed not only mild deficits of memory and concentration but also severe deficits in 
inmmediate memory and difficulties in planning and consolidation of newly learned information, all of which are hardly explainable only because of the neuropsychological effects of long lasting compensated hydrocephalus. As an example, D'Esposito et al described a patient with a traumatic penetrating brain injury which affected exclusively the fornix, and where the consequences were persistent anterograde amnesia. ${ }^{15}$ Moreover, Damasio et al reported several injuries in the frontal-basal areas of the brain-the septal nucleus, the acumbens nucleus, the innominated substance, and the connecting passages - with important memory difficulties and personality changes. These may explain the memory problems caused by the dysfunction in the hippocampal system, less severe than the injury in the above mentioned areas and simultaneously associate this dysfunction with a possible specific reduction in cholinergic neurotransmitters. ${ }^{17}$ Recently, the relation between the ventral hippocampus and its nicotinic receptors and memory has been described. ${ }^{18}$

On the other hand, the hypothalamus regulates such important physiologically based drives as appetite, sexual arousal, and thirst. Depending on the site of the damage, lesions of hypothalamic nuclei can result in various symptoms, including obesity, disorders of temperature control, and diminished drives states and responsivity. ${ }^{19}$ Mood states may also be affected by hypothalamic lesions. ${ }^{20}$ The hypothalamus is also involved in vegetative and protective drive states, movement patterns, and associated emotional responses, due to its connections with the limbic system.

In summary, we have described a severe psychiatric complication after endoscopic third ventriculostomy which allowed us to locate specific neuropsychological dysfunctions attributable to lesions to the frontal-basal areas of the brain, the hypothalamus, the limbic system, and its connections. Clinicians should take this potential complication of endoscopic third ventriculostomy into account before recommending this so-called minimally invasive procedure.

1 Organización Mundial de la Salud. CIE 10. Décima revisión de la Clasificación Internacional de Enfermedades. Trastornos mentales y del comportamiento. Descripciones clínicas y pautas para el diagnóstico. Madrid: Meditor, 1992.

2 American Psychiatric Association. Diagnostic and statistical manual of mental disorders. 4th ed. Washington DC: APA, 1994

3 Kaplan HI, Sadock BJ, Crebb JA. Synopsis of psychiatry. 7th ed. Baltimore: Williams and Wilkins, 1994.

4 Vries JK. An endoscopic technique for third ventriculostomy. Surg Neurol 1978;9:165-8.

5 Viñas FC, Dujovny N, Dujovny M. Microanatomical basis for the third ventriculostomy. Minim Invasive Neurosurg for the third ventris

6 Wechsler D. Wechsler adult intelligence scale. New York: The Psychological Corporation, 1955

7 Wechsler D. Wechsler memory scale-revised manual. New York. The Psychological Corporation Harcourt Brace Jovanovich, 1987.

8 Reitan RM, Wolfson D. The Halstead-Reitan neuropsychological test battery: theory and clinical interpretation. Tucson: Neuropsychology Press, 1985.

9 Harlow JM. Passage of an iron rod through the head. Boston Medical and Surgical fournal 1949;39:389.

10 Harlow JM. Recovery from the passage of an iron bar Harlow JM. Recovery from the passage of an iron bar
through the head. Publications of the Massachusetts Medical Society 1968;2:327-47.

11 Damasio AR. Descartes' error: emotion, reason, and the human brain. London: Papermac, 1996.

12 Brockmeyer D, Abtin K, Carey L, et al. Endoscopic third entriculostomy: an outcome analysis. Pediatr Neurosurg 1998;28:236-40

13 Baskin JJ, Manwaring KH, Rekate HL. Ventricular shunt removal: the ultimate treatment of the slit ventricle syndrome. F Neurosurg 1998;88:478-84.

14 Labbate LA, Warden D, Murray GB. Salutary change after frontal brain trauma. Ann Clin Psychiatry 1997; 9:27-30.

15 Abe K, Inokawa M, Kashiwagi A, et al. Amnesia after a discrete basal forebrain lesion. F Neurol Neurosurg Psychiatry crete basal forebrain

16 D'Esposito M, Verfaellie M, Alexander MP, et al. Amnesia following traumatic bilateral fornix transection. Neurology 1995;45:1546-50.

17 Damasio AR, Graff-Radford NR, Eslinger PJ, et al. Amnesia following basal forebrain lesions. Arch Neurol 1985;42:26371 .

18 Levin DE, Christopher NC, Weaver T, et al. Ventral hippocampal ibotenic acid lesions block chronic nicotine induced spatial working memory improvement in rats. Brain Res Cogn Brain Res 1999;7:405-10.

19 Flynn FG, Cummings JL, Tomiyasu U. Altered behavior associated with damage to the ventromedial hypothalamus: a distinctive syndrome. Behavioural Neurology 1988;1:4958 .

20 Shepherd GM. Neurobiology. 2nd ed. New York: Oxford University Press, 1988 